The complicated connection between the parameter of intensity, spectral dependence, temperature and cathode quality can only be measured in stages, and for the time being no statement on the nature of the anomalies is available, apart from the fact that they have now been identified definitely as discontinuities. Further development of the results now obtained will probably benefit from the prospect of relationships in solid state physics being clarified on the lines of the assumption made by SeIrz which was mentioned at the outset. Also useful should be a significant advance, in the form of a purely electronic high-sensitivity radiation detector, into spectral regions that have been virtually inaccesible till now.

\section{Acknowledgements}

We should like to express our appreciation to Prof. W. Heimans, Wiesbaden, for providing the photoelectric cells, to Prof. A. Schlürer, Munich, for valuable advice and to Dr. P. Tнома for making the measuring equipment available.

\title{
Kombination von galvanomagnetischen- und Feldeffekt-Messungen an Germanium-Bikristallen
}

\author{
G. LANDWEHR * \\ Mitteilung aus der Physikalisch-Technischen Bundesanstalt \\ (Z. Naturforschg. 20 a, 1599-1611 [1965]; eingegangen am 24. August 1965)
}

\begin{abstract}
The transport properties in a magnetic field of the p-type space charge region adjacent to $20^{\circ}$ tilt grain boundaries in n-type germanium bicrystals were investigated as a function of a reverse bias voltage between bulk and grain boundary (field effect). The average $\mathrm{H}_{\text {ALL }}$ mobility at $77^{\circ} \mathrm{K}$ of the holes decreased with increasing bias, demonstrating the existence of a size effect caused by diffuse boundary scattering in connection with the small width of the space charge layer. The results obtained for symmetrical bias are in agreement with a simplified theory based on a constant space charge field and allow to estimate an average hole density. The transverse magnetoresistance also decreased with increasing field effect-voltage, as expected.
\end{abstract}

Als Korngrenze bezeichnet man eine Fläche in einem Festkörper, an der zwei einkristalline Bereiche einander berühren. Es ist lange bekannt, daß Korngrenzen die elektrische Leitfähigkeit von Metallen und Halbleitern ${ }^{1}$ beeinflussen können. Seitdem gezeigt wurde ${ }^{2,3}$, daß es möglich ist, durch Ziehen von Germanium-Bikristallen mit vorgegebenem Verdrehungswinkel ebene Korngrenzen großer Ausdehnung herzustellen, ist eine Reihe von Arbeiten erschienen, die sich mit dem Stromtransport in diesem Material parallel und senkrecht zu Korngrenzen befassen ${ }^{3-8}$. Die Auswirkungen von Korngrenzen (in Bikristallen mit nicht zu kleinem Verdrehungswinkel) in n-leitendem Germanium sind besonders markant, da sie sich mit einer dünnen, p-leitenden Raumladungszone umgeben, und somit Anlaß zu einer Po-

* Braunschweig.

1 W. E. Taylor, N. H. Odell u. H. Y. Fan, Phys. Rev. 88, 867 [1952].

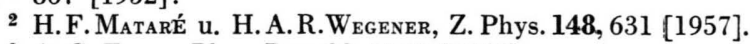

3 A. G. Tweet, Phys. Rev. 99, 1182 [1955].

4 B. Reed, O. A. Weinreich u. H. F. Mataré, Phys. Rev. 113, 454 [1959].

5 G. Landwehr u. P. Handler, J. Phys. Chem. Solids 23, 891 [1962]. tentialstufe sind. Die Ursache für die Defektleitung an der Korngrenze ist gegenwärtig nicht mit Sicherheit bekannt. Vieles deutet darauf hin, daß der Akzeptorcharakter von Versetzungen herrührt, die nicht abgesättigte chemische Bindungen aufweisen. Überlegungen, die auf der Versetzungstheorie ${ }^{9}$ beruhen, führen zu dem Schluß, daß (bei Voraussetzung eines einfachen kubischen Gitters) ein Bikristall, dessen Hälften gegeneinander verdreht und nicht verschraubt sind, dann mechanisch am stabilsten ist, wenn in der Berührungsebene eine regelmäßige Anordnung von Stufenversetzungen vorhanden ist. Dieses Konzept wird bei Verdrehungswinkeln, die größer als ca. $15^{\circ}$ sind, problematisch; außerdem sind die Verhältnisse beim Diamantgitter komplizierter. Hornstra ${ }^{10}$ hat jedoch gezeigt, daß beim Germa-

${ }^{6}$ Y. Hamakawa u. Y. Yamaguchi, Jap. J. Appl. Phys. 1, 334 [1962].

7 Y. Matukura, J. Phys. Soc. Japan 17, 1405 [1962].

8 R. K. Müller, J. Appl. Phys. 32, 635 [1961].

9 Siehe z. B. W. T. Read JR., Dislocations in Crystals, McGraw-Hill Book Co., New York 1953.

10 J. Hornstra, Physica 26, 198 [1960]. 
nium auch bei großen Fehlorientierungen das Versetzungsmodell einer Korngrenze noch sinnvoll ist. Sowohl Anordnungen mit gesättigten als auch solche mit ungesättigten Bindungen scheinen möglich. Freie Valenzen haben die Tendenz, sich abzusättigen und sind als Ursache für die Akzeptoreigenschaften von Versetzungen denkbar. Diese Vorstellung (dangling bonds) stammt von SHockLEY ${ }^{11}$.

Andererseits kann grundsätzlich nicht ausgeschlossen werden, daß sich Fremdatome mit Akzeptorcharakter an den Versetzungen abgeschieden haben. Die Frage, ob freie Bindungen für die Defektleitung von Korngrenzen verantwortlich sind, soll hier nicht weiter diskutiert werden, da sie im Hinblick auf das Ziel dieser Arbeit nicht von Bedeutung ist.

Verschiedene Befunde deuten darauf hin, daß die stromführende Raumladungszone an der Korngrenze von Germanium-Bikristallen mit einem Verdrehungswinkel von $20^{\circ}$ sehr dünn ist. Eine Analyse von Leitfähigkeits- und HALL-Effekt-Daten ${ }^{12}$ führt zu dem Ergebnis, daß bei Temperaturen des flüssigen Heliums die Dicke der stromtragenden Schicht von der Größenordnung $100 \AA$ sein dürfte. Von dünnen Metallschichten ${ }^{13}$ und Inversionsschichten an oxydierten Halbleiteroberflächen ${ }^{14}$ ist bekannt, daß die Beweglichkeit der Ladungsträger geringer ist als im kompakten Material, wenn die Dicke der Schicht geringer ist als die mittlere freie Weglänge im Volumen. Das bedeutet, daß (bei isotroper Beweglichkeit im Grundmaterial) die Zusammenstöße mit der Oberfläche unelastisch sind und die Beweglichkeit reduzieren. Eine Korngrenze in Germanium sollte ähnlich streuen wie eine reale Oberfläche, so daß Weglängeneffekte zu erwarten sind. Eine früher beobachtete ausgeprägte Anisotropie ${ }^{5}$ in der Winkelabhängigkeit der transversalen magnetischen Widerstandsänderung legt den Schluß nahe, daß diffuse Streuung an der Korngrenze eine Rolle spielt.

Es erschien deshalb wünschenswert, diese Weglängeneffekte genauer zu studieren. Eine Möglichkeit dazu bieten Experimente ${ }^{14,15}$, die auf der gleichen Grundlage beruhen, wie diejenigen an „channels" auf Halbleiteroberflächen. Beispielsweise kann man über der Basis eines pnp-Transistors durch Ein-

11 W. Shockley, Phys. Rev. 91, 228 [1953].

12 G. Landwehr, Phys. Status Solidi 3, 440 [1963].

13 Siehe z. B. J. M. Zrman, Electrons and Phonons, Oxford University Press, London 1960, S. 451.

14 W. L. Brown, Phys. Rev. 91, 518 [1953].

15 H. Statz, G. A. de Mars, L. Davis Jr. u. A. Adams Jr., Phys. Rev. 101, 1272 [1956]. bringen in eine passende Atmosphäre eine dünne, p-leitende Inversionsschicht erzeugen, für die sich die Bezeichnung „channel“ eingebürgert hat. Legt man an den pn-Übergang channel - Basis eine Sperrspannung an, so ändert sich die Leitfähigkeit der Inversionsschicht. Diese Erscheinung wird vielfach als Feldeffekt bezeichnet. Theoretische Überlegungen ${ }^{16}$ führen zu dem Schluß, daß das Oberflächenpotential beim Feldeffekt im wesentlichen konstant bleibt und daß die Beweglichkeit der Ladungsträger in der Oberflächenschicht mit wachsender Sperrspannung abnimmt, als Folge der Einschnürung des Strompfades und diffuser Streuung an der Oberfläche.

Bei Feldeffekt-Experimenten an Bikristallen wird eine negative Vorspannung zwischen der p-Zone und dem Kristalläußeren angelegt. Das führt zu einer erhöhten Feldstärke in der Raumladungszone und zu einer Abnahme der Dicke der stromführenden pSchicht. Da die Lage der Fermi-Kante am Orte der Korngrenze nur ungenau bekannt ist, und da die existierenden Theorien zum Feldeffekt an Oberflächeninversionsschichten nur bedingt auf das analoge Problem bei einer Korngrenze angewendet werden können, wurde eine möglichst direkte experimentelle Bestimmung der Löcherbeweglichkeit in der Raumladungszone als Funktion der angelegten Vorspannung angestrebt. Zu diesem Zweck wurden bei konstanter Temperatur HALL-Effekt und elektrische Leitfähigkeit der p-Schicht bei verschiedenen Vorspannungen $V_{\mathrm{F}}$ gemessen, und damit die Hall-Beweglichkeit in Abhängigkeit von $V_{\mathrm{F}}$. Dabei zeigte sich, daß - wie erwartet - die HALL-Beweglichkeit mit wachsender Vorspannung sinkt.

Darüber hinaus wurde auch die magnetische Widerstandsänderung als Funktion einer zwischen Korngrenze und Kristalläußerem angelegten Spannung untersucht. Über einen Teil der Ergebnisse wurde bereits kurz berichtet ${ }^{17}$.

\section{Experimentelles}

a) Proben

Die Mehrzahl der Versuchsproben wurde aus einem in Abb. 1 schematisch dargestellten Bikristall ${ }^{17 a}$ präpariert, der die gleiche Fehlorientierung hatte wie frü-

16 J. R. Schrieffer, Phys. Rev. 97, 641 [1955].

17 G. Landwehr, Z. Naturforschg. 19 a, 157 [1964].

${ }^{17}$ a Der Kristall wurde freundlicherweise von Herrn Dr. P. H. KEсK von den General Telephone and Electronics Laboratories zur Verfügung gestellt. 
her untersuchte Bikristalle, d.h. die [001]-Richtungen der Kristallhälften waren $10^{\circ}$ symmetrisch um eine [100]-Achse verdreht, so daß der Gesamtdrehwinkel $20^{\circ}$ betrug. Die Versuchsexemplare wurden so geschnitten, daß ihre Längsachsen zur Ziehrichtung parallel waren. Somit verliefen die Stufenversetzungen in der Korngrenze senkrecht zur Probenachse, s. Abb. 1. Das

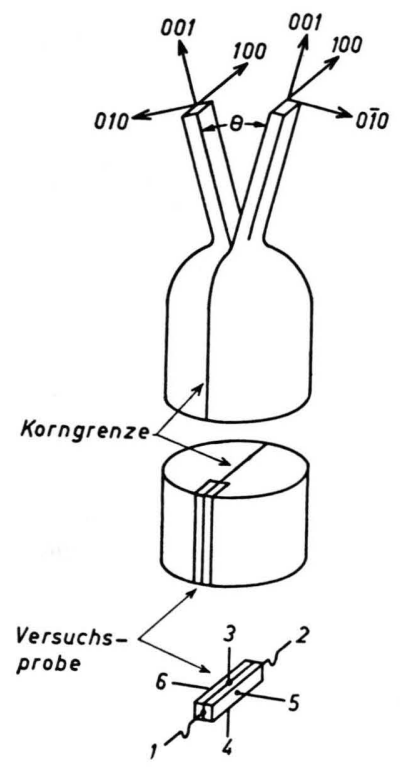

\begin{abstract}
Abb. 1. Schematische Darstellung des Bikristalls. Der Verdrehungswinkel $\Theta$ beträgt $20^{\circ}$. Weiterhin ist die Schnittrichtung der Versuchsproben angezeigt sowie die Lage der Kontakte. 1-2: Stromkontakte, 3-4: HaLL-Kontakte, 5-6: Feldeffektsonden.
\end{abstract}

Kristallgrundmaterial war vom $\mathrm{n}$-Typ. Dadurch wurde eine elektrische Trennung von Raumladungszone und Kristalläußerem möglich. Durch Anlegieren von kleinen Indium-Kugeln an die Korngrenzen-Region wurden „ohmsche“ Kontakte mit der Raumladungszone und gleichrichtende mit dem Kristalläußeren gebildet. Beim Anlegen einer Spannung an die Stromkontakte der Versuchsproben fließt praktisch der gesamte Strom durch die dünne $\mathrm{p}$-Zone, da - unabhängig von der Polung stets einer der pn-Übergänge sperrt und einen Widerstand hat, der sehr groß ist gegenüber demjenigen der Raumladungsschicht, deren Eigenschaften erforscht werden sollen. Der spezifische Widerstand des Grundmaterials der Versuchsproben konnte sich von einem Ende zum anderen maximal von $2,8 \Omega \mathrm{cm}$ auf $3,4 \Omega \mathrm{cm}$ ändern. Diese Inhomogenität kann als unwesentlich im Hinblick auf das Ziel dieser Untersuchungen angesehen werden. Zwei Proben (N 8 und N 9) mit den Dimensionen von etwa $12 \times 1,5 \times 1,5 \mathrm{~mm}^{3}$ dienten dazu, die Temperatur-, Winkel- und Feldstärkeabhängigkeit der galvanomagnetischen Größen zu messen. Sie wurden, in der Weise wie bereits beschrieben ${ }^{5}$, mit 6 Indium-Kontakten versehen. An Probe N 8 wurden zusätzlich 2 Feldeffektelektroden angebracht. Bei drei Kristallen (Abmessungen etwa $11 \times 1,5 \times 1 \mathrm{~mm}^{3}$ ), die vorwiegend zu Feldeffektmessungen dienten, wurde eine unterschiedliche Elektrodenkonfiguration benutzt. Wie aus Abb. 1 ersichtlich, wurde auf getrennte Strom- und Potentialsonden verzichtet. Das ist möglich, weil der Wi- derstand der Korngrenze relativ hoch ist und der Kontaktwiderstand vernachlässigbar klein. Zwei HALL-Kontakte wurden etwa in der Mitte der Probe anlegiert. Die Sonden zum Anlegen der Vorspannung waren Druckkontakte aus Golddraht, die in einigen Fällen mit Silberpaste fixiert waren. Die Feldkontakte wurden an beiden Seiten der Bikristalle angebracht, so daß die Korngrenze symmetrisch oder asymmetrisch vorgespannt werden konnte. Um störende Oberflächeneffekte zu vermeiden, wurden alle Versuchsexemplare sorgfältig mit CP 4 geätzt. Alle Zuleitungen bestanden aus Golddraht und waren in eine Probenhalterung aus Kunststoff so eingeklebt, daß keine mechanischen Verspannungen auftreten konnten. Die Verwendung einer derartigen Halterung ermöglichte ein Ätzen der Kristalle auch nach der Montage. Zur Kontrolle der Messungen wurde eine Probe aus einem früher untersuchten ${ }^{5} 2 \Omega \mathrm{cm}$-Bikristall vom n-Typ präpariert, mit den Versetzungen parallel zur Stromrichtung.

\section{b) Meßtechnik}

Zur Erzeugung tiefer Temperaturen diente ein konventionelles Doppel-Dewar-System aus Glas, das in Abb. 2 skizziert ist. Konstante Temperaturen zwischen 4,2 und $1,15^{\circ} \mathrm{K}$ konnten durch Abpumpen von Helium im inneren Gefäß erzeugt werden, unter Verwendung

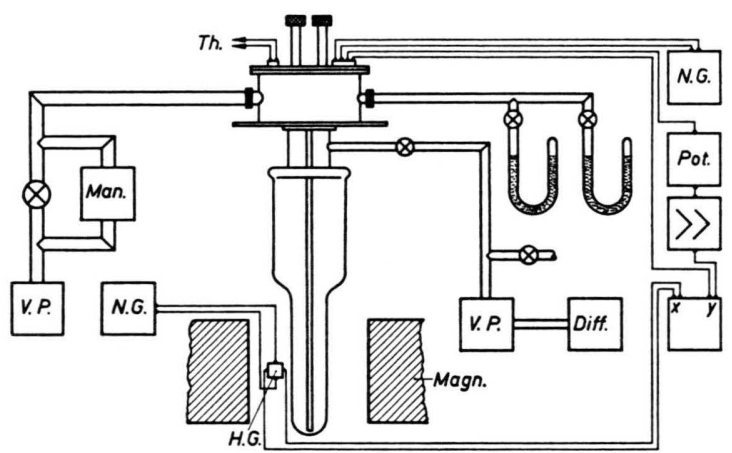

Abb. 2. Schema der Versuchsanordnung.

V.P. $=$ Vorpumpe, Man. $=$ Manostat, N.G.=Gleichstrom-Netzgerät, H.G. = InAs-HALL-Generator, Magn.=12" Varian Magnet, Diff. = Diffusionspumpe, $\mathrm{XY}=$ Koordinatenschreiber, Pot. $=$ Gleichstromkompensator, Th. $=$ Thermometer-Zuleitungen.

eines Metall-Manostaten. Die Versuchsproben tauchten direkt in das flüssige Helium ein. Wenn Experimente bei Stickstoff-Temperaturen ausgeführt wurden, waren die Proben entweder in unmittelbarem Kontakt mit flüssigem $\mathrm{N}_{2}$, oder nur das äußere Dewar-Gefäß war mit Stickstoff gefüllt. Die Kristalle befanden sich dann in einer Atmosphäre aus Stickstoff- oder Helium-Gas und der Druck zwischen den Wänden des inneren Dewar-Gefäßes wurde so hoch eingestellt, daß ein guter Wärmekontakt gewährleistet war. Im letzteren Fall wurde die Temperatur der Versuchsprobe mit einem Kupfer-Konstantan-Thermoelement gemessen, das in unmittelbarer Nähe der Bikristallprobe montiert war. Bei Helium-Temperaturen wurde ein Dampfdruckther- 
mometer in Verbindung mit einem $50 \Omega$-A lle n B rad le y - Kohlewiderstand benutzt. Der Kryostat war zwischen den Polschuhen eines $12^{\prime \prime} \mathrm{V}$ a ri a n-Magneten montiert, der $360^{\circ}$ um seine vertikale Achse rotiert werden konnte. Bei einem Polschuhabstand von $3^{\prime \prime}$ betrug das maximal erreichbare Magnetfeld 10,5 kOe. An einem der Magnet-Pole war ein mit Protonenkernresonanzen kalibrierter InAs-HALL-Generator befestigt, der eine dem Feld proportionale Spannung lieferte. Zur Messung der Feldabhängigkeit des HALL-Effektes oder der magnetischen Widerstandsänderung der p-Zone (bei konstanter Temperatur) wurde die HALL-Spannung der InAs-Sonde auf den $X$-Eingang eines $\mathrm{M}$ os ele y Koordinaten-Schreibers gegeben. Durch die Versuchsexemplare wurde ein konstanter Strom der Größenordnung 2 bis $10 \mu \mathrm{A}$ geschickt und der größte Teil des entstehenden Spannungsabfalls bei der Messung der magnetischen Widerstandsänderung mit einem Kompensator subtrahiert. Die Spannungs-Differenz wurde über einen empfindlichen Verstärker (H e w le t t $\mathrm{P}$ a ck a rd 425 A) dem $Y$-Eingang des Schreibers zugeleitet. Durch langsames Steigern des Magnetfeldes von Null auf den Endwert erhält man die gewünschten Daten rasch, kontinuierlich und mit großer Genauigkeit. Analoges gilt für den HaLL-Effekt. Die Methode ist besonders nützlich für Messungen bei kleinen Feldstärken. In gleicher Weise lassen sich die galvanomagnetischen Größen messen, wenn das Kristalläußere mittels der Feldeffektelektroden gegenüber der p-Schicht an der Korngrenze in Sperrichtung vorgespannt ist. Es wurde darauf geachtet, daß der Sperrstrom des pnÜbergangs Korngrenze-n-Kristall sehr klein war gegenüber dem Probenlängsstrom, damit die Meßergebnisse nicht verfälscht wurden.

Der Feldeffekt, d.h. die Widerstandsänderung der Korngrenzenregion als Funktion der angelegten Vorspannung, wurde in der Regel bei konstantem Strom mit einem Kompensator gemessen. Vor Beginn der Untersuchungen wurden mit Hilfe eines Koordinatenschrei-

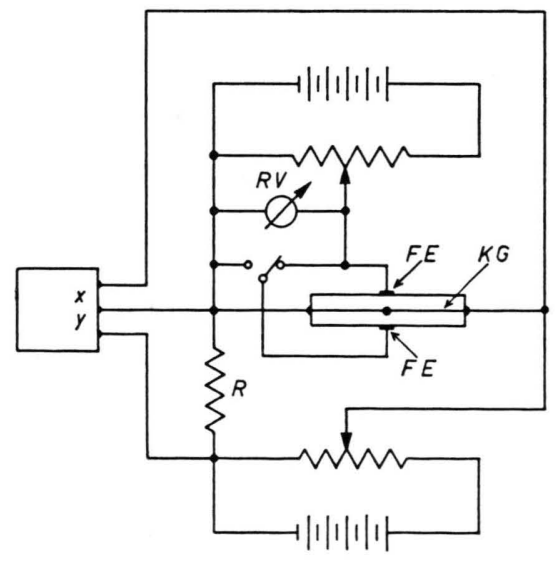

Abb. 3. Schaltung zur Aufnahme von Strom-Spannungs-Kennlinien von Bikristallen bei verschiedenen Vorspannungen zwischen Raumladungszone und Kristallgrundmaterial. Es bedeuten: xy: Koordinatenschreiber, R: Meßwiderstand, FE: Feldeffekt-Sonden, KG: Korngrenze, RV: Röhrenvoltmeter. bers in einer Schaltung gemäß Abb. 3 jeweils StromSpannungskennlinien mit der Vorspannung als Parameter aufgenommen. Der Grund für dieses Verfahren liegt in der Notwendigkeit, einen Spannungsbereich abzugrenzen, in dem der Strom linear von der Spannung längs der Korngrenze abhängt. Bei den benutzten Proben ist das mit hinreichender Näherung nur unterhalb von etwa $0,5 \mathrm{~V}$ der Fall. In Abb. 4 ist eine bei $4,2{ }^{\circ} \mathrm{K}$ aufgenommene Schar von Strom-Spannungscharakteristiken dargestellt, mit der an eine Kristallhälfte gelegten Vorspannung als Parameter.

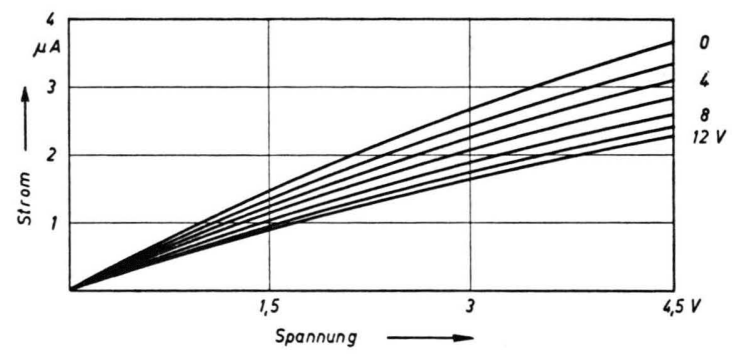

Abb. 4. Strom parallel zur Korngrenze als Funktion der angelegten Spannung mit der Potentialdifferenz zwischen pZone und einer Kristallhälfte als Parameter. Probe IV bei $T=4,2^{\circ} \mathrm{K}$.

Es ist ersichtlich, daß bei höheren Spannungen der differentielle Widerstand wächst. Dieses Verhalten wird durch einen internen Feldeffekt verursacht, der dadurch zustande kommt, daß die p-Zone gegenüber dem Kristall vorgespannt ist. Während das Potential in der Korngrenzenschicht von einem Ende der Probe zum anderen linear ansteigt, ist der Spannungsabfall im n-Material praktisch gleich Null, da fast die gesamte Spannung an dem in Sperrichtung gepolten Kontakt liegt. Dadurch entsteht eine ortsabhängige Vorspannung zwischen Korngrenze und Kristalläußerem, die qualitativ wie eine extern angelegte Spannung zwischen den Feldelektroden wirkt. Es wurde dafür Sorge getragen, daß die äußere Vorspannung stets groß war gegenüber der ,internen“.

Mit der Anordnung nach Abb. 3 lassen sich weiterhin zu große Sperrströme zwischen n-Material und pZone leicht feststellen. Bei der Längsspannung Null muß beim Anlegen der Vorspannung der einmal eingestellte Nullpunkt erhalten bleiben. Die Größe des Sperrstromes als Funktion der Vorspannung wurde bei jedem Versuch gemessen. Ferner wurde sichergestellt, daß die Durchschlagspannung der Strom- und HALLKontakte hinreichend groß $(>60 \mathrm{~V})$ war. Bei der Messung der Temperaturabhängigkeit der galvanomagnetischen Effekte der Proben N 8 und N 9 wurde eine bereits beschriebene Technik ${ }^{5}$ angewandt.

\section{Ergebnisse}

Die galvanomagnetischen Messungen an den Proben $\mathrm{N} 8$ und $\mathrm{N} 9$ wurden vorgenommen mit dem Ziel, Gewißheit darüber zu schaffen, daß die Eigen- 
schaften des Bikristalls mit denen von früher untersuchten Kristallen in Einklang sind. Das war, wie erwartet, der Fall. Die Leitfähigkeit pro Flächenstück

$$
\sigma_{0}=S \cdot l / h
$$

( $S=$ Leitfähigkeit, $l=$ Länge der Probe, $h=$ Höhe der Probe) der p-Schicht zwischen 4,2 und $20^{\circ} \mathrm{K}$ war praktisch konstant und betrug etwa $1,5 \cdot 10^{-4}$ $\Omega^{-1}$. Sie lag damit niedriger als bei vorher untersuchten Exemplaren, bei denen für $\sigma_{0}$ im Temperaturgebiet des flüssigen Heliums ein Wert von rund $2,3 \cdot 10^{-4} \Omega^{-1}$ gefunden wurde. Es ist zweckmäßig, die Leitfähigkeit pro Flächeneinheit der Korngrenzenebene anzugeben, da die Dicke der stromführenden p-Schicht nicht genau bekannt ist. Analoges gilt für den HaLL-Koeffizienten. Der reduzierte HaLLKoeffizient $R^{*}$ ist definiert als

$$
R^{*}=V_{\mathrm{H}} / I H=R / t
$$

mit $V_{\mathrm{H}}=$ HaLL-Spannung, $I=$ Stromstärke, $H=$ magnetische Feldstärke, $R=$ Volumen-HaLL-Koeffizient, $t=$ mittlere Dicke der stromführenden p-Schicht. Bei Vorhandensein von nur einer Art von Ladungsträgern ist $R^{*}$ ein $\mathrm{Maß}$ für die Flächenladungsdichte $p$ der Defektelektronen, es gilt dann (bei starken Magnetfeldern) $R^{*}=1 / p e, e=$ Elementarladung. Das Produkt $R^{*} \sigma_{0}$ ist unabhängig von $t$ und wird als mittlere HALL-Beweglichkeit bezeichnet. Für $R^{*}$ von Probe N 8 wurde im überstrichenen Bereich von $1000-10000$ Oe ein konstanter Wert von $8 \cdot 10^{5}$ $\mathrm{cm}^{2} / \mathrm{C}$ gefunden. Das ist etwa $30 \%$ weniger als früher gemessen. Die Ursache der Abweichungen ist nicht bekannt. Die Temperaturabhängigkeit von $\sigma_{0}$

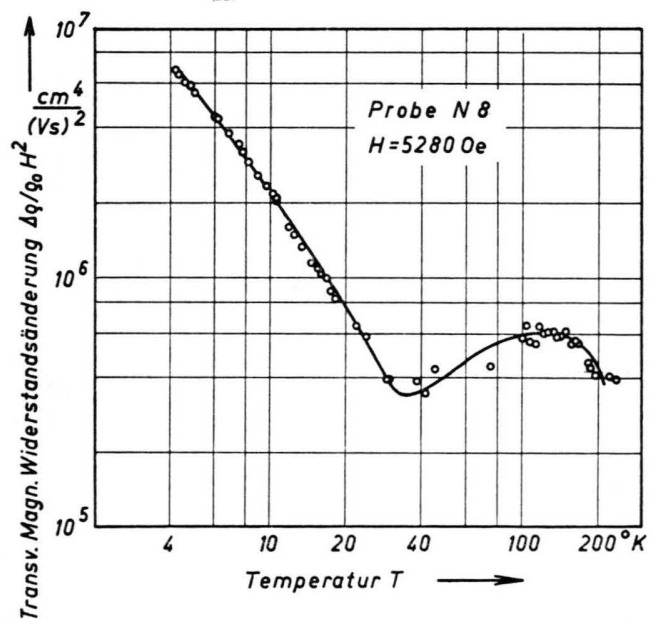

Abb. 5. Abhängigkeit der transversalen magnetischen Widerstandsänderung ( $H \perp$ Korngrenze) von der Temperatur. und $R^{*}$ von Probe $\mathrm{N} 8$ bei höheren Temperaturen war im übrigen qualitativ die gleiche wie bei vorher untersuchten Bikristallproben aus $2 \Omega \mathrm{cm}$ Grundmaterial vom n-Typ, d. h. $\sigma_{0}$ und $R^{*}$ weisen ein flaches Maximum bei 150 bzw. $120^{\circ} \mathrm{K}$ auf, mit $\sigma_{0}=2,7 \cdot 10^{-4} \Omega^{-1}$ und $R^{*}=1,35 \cdot 10^{6} \mathrm{~cm}^{2} / \mathrm{C}$ bei $77^{\circ} \mathrm{K}$. In Abb. 5 ist die Temperaturabhängigkeit der transversalen magnetischen Widerstandsänderung mit $H$ senkrecht zur Korngrenze aufgetragen. Die Übereinstimmung mit früheren Messungen ist gut. Auch die Abhängigkeit der transversalen magnetischen Widerstandsänderung vom Magnetfeld bei $4,2{ }^{\circ} \mathrm{K}$ ist praktisch identisch mit älteren Ergebnissen. Für Probe N9 ist in Abb. 6 die Winkelabhängigkeit der transversalen magnetischen Widerstandsänderung bei $77^{\circ} \mathrm{K}$ dargestellt. Wenn das

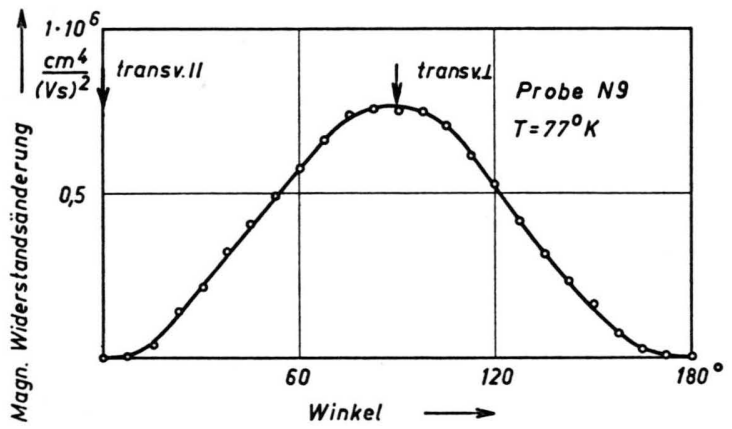

Abb. 6. Winkelabhängigkeit der transversalen magnetischen Widerstandsänderung. Es bedeuten: transv. $\| H$ parallel zur Korngrenze, transv. $\perp H$ senkrecht zur Korngrenze.

Magnetfeld parallel zur Korngrenze zeigt, ist die Widerstandsänderung um mehr als 2 Größenordnungen kleiner als im Falle eines zur Korngrenzenebene senkrechten Feldes. Die starke Anisotropie kann als Beweis dafür gewertet werden, daß die stromführende Zone sehr dünn ist und daß Leckströme im Volumen nicht von Bedeutung sind.

Aus der Gesamtheit der ausgeführten Messungen an den Proben N 8 und N 9 läßt sich der Schluß ziehen, daß der Bikristall, aus dem die Versuchsexemplare für die Feldeffektexperimente hergestellt wurden, Eigenschaften aufweist, die an einer größeren Zahl von Proben gleicher Fehlorientierung gefunden wurden. Die Abweichungen in den numerischen Werten von $R^{*}$ und $\sigma_{0}$ sind nicht so groß, da $\beta$ sie bedenklich wären. Um jedoch sicher zu sein, daß der untersuchte Bikristall keine Ausnahmestellung einnimmt, wurde eine Feldeffektprobe aus einem anderen $2 \Omega \mathrm{cm}$-Bikristall mit bekannten Eigenschaften präpariert. 
Im folgenden sollen Resultate berichtet werden, die bei Experimenten mit der Feldeffektprobe IV erzielt wurden und die für die untersuchten Bikristallproben typisch sind. In Abb. 7 ist die relative Widerstandsänderung als Funktion der Vorspannung

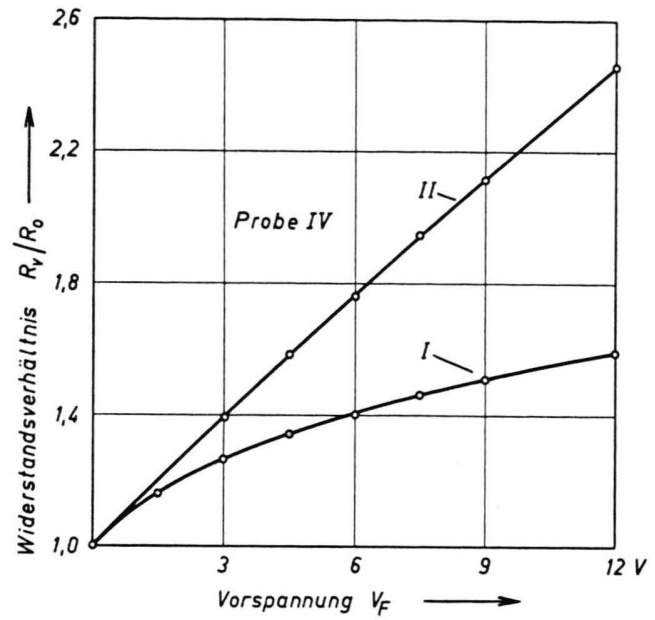

Abb. 7. Relative Widerstandsänderung $R_{\mathrm{v}} / R_{0}$ der BikristallRaumladungszone als Funktion der Vorspannung $V_{\mathrm{F}}$ bei $T=77^{\circ} \mathrm{K}$.

$V_{\mathrm{F}}$ aufgetragen. Ist nur eine Feldeffektelektrode vorgespannt (und die andere mit einer der Stromzuführungen verbunden), wächst der Widerstand gemäß Kurve I an, bei Parallelschaltung der Feldsonden ist die relative Widerstandserhöhung größer, bei einer Spannung von $12 \mathrm{~V}$ etwa 2,5-fach, siehe Kurve II. Die Werte wurden bei einer Temperatur von $77^{\circ} \mathrm{K}$ gemessen. Die Leitfähigkeit pro Flächenstück bei $V_{\mathrm{F}}=0$ betrug $2,52 \cdot 10^{-4} \Omega^{-1}$ in Übereinstimmung mit Messungen an Probe N 8. Der Strom durch die Probe hatte die Größe 4,14 $\mu$ A. Der Rückstrom der in Sperrichtung gepolten, parallel geschalteten Feldsonden betrug $0,4 \mathrm{~m} \mu \mathrm{A}$ bei $V_{\mathrm{F}}=3 \mathrm{~V}$ und $7,4 \mathrm{~m} \mu \mathrm{A}$ bei $V_{\mathrm{F}}=9 \mathrm{~V}$ und war damit zu vernachlässigen. Bei den Sperrströmen handelte es sich offenbar um Oberflächenströme, die durch Ausheizen des Versuchskristalls vor der Abkühlung noch herabgesetzt werden konnten. Eine zu starke Reduzierung war jedoch nicht praktisch, da bei sehr kleinen Rückströmen (vor allem bei kleinen Vorspannungen) zu viel Zeit zur Gleichgewichtseinstellung nach einer Änderung von $V_{\mathrm{F}}$ gebraucht wurde. Sehr kleine Sperrströme führten deshalb zu einer Vergrößerung der Meßunsicherheit und nicht zu einer Herabsetzung, wie man zunächst vermuten würde.
Das Ergebnis einer HaLL-Effekt-Messung in Abhängigkeit von der Vorspannung bei $T=77^{\circ} \mathrm{K}$ ist in Abb. 8 dargestellt. Der reduzierte $\mathrm{H}_{\mathrm{ALL}-\mathrm{Koeffi}}$ zient $R^{*}$ wächst mit größer werdendem $V_{\mathrm{F}}$. Sein Wert bei $V_{\mathrm{F}}=0$ von $R^{*}=1,36 \cdot 10^{6} \mathrm{~cm}^{2} / \mathrm{C}$ (unabhängig von $H$ bis zu $10 \mathrm{kOe}$ ) stimmt gut mit dem von Probe N 8 überein. Es ist bemerkenswert, daß

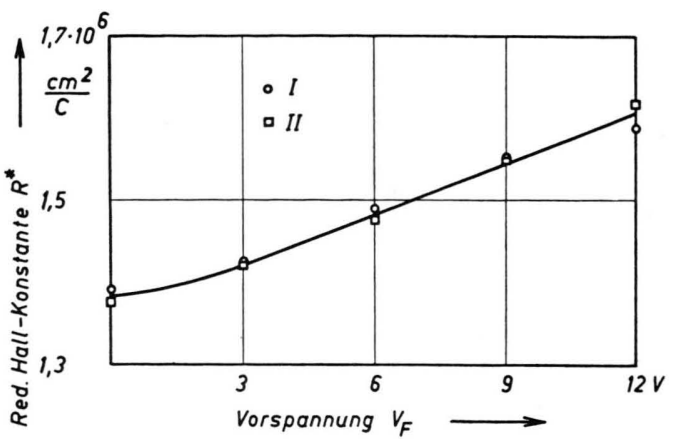

Abb. 8. Reduzierte Hall-Konstante $R^{*}$ als Funktion der Vorspannung bei $77^{\circ} \mathrm{K}$. I= asymmetrische Vorspannung, $\mathrm{II}=$ symmetrische Vorspannung.

der Anstieg von $R^{*}$ für symmetrische und unsymmetrische Vorspannung der Korngrenze innerhalb der Meßunsicherheit von etwa $\pm 2 \%$ gleich groß ist. Beim Anlegen einer Vorspannung von $12 \mathrm{~V}$ nimmt $R^{*}$ um ca. $17 \%$ zu. Ein Vergleich mit Abb. 7 zeigt, daß der größte Teil des Widerstandsanstiegs beim Anlegen einer Vorspannung durch eine Beweglichkeitsabnahme zustande kommen muß. Quantitativ kommt dies in Abb. 9 zum Ausdruck, in der

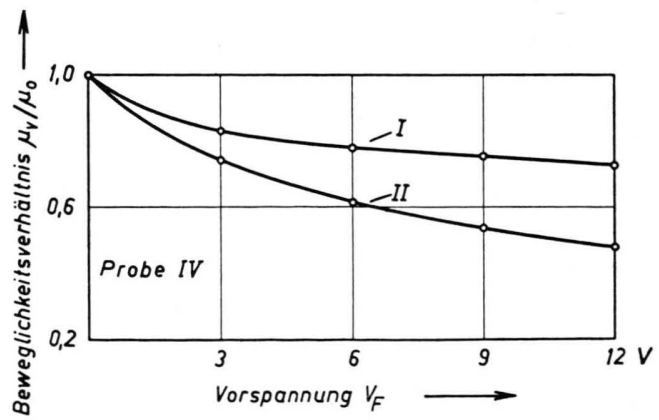

Abb. 9. Relative Änderung der Hall-Beweglichkeit mit der Vorspannung bei asymmetrischer (I) und symmetrischer Vorspannung (II) der Raumladungszone gegenüber dem Kristalläußeren.

die relative Änderung der $\mathrm{H}_{\text {ALL-Beweglichkeit }}$ $\left(R^{*} \sigma_{0}\right)_{\mathrm{V}} /\left(R^{*} \sigma_{0}\right)_{0}$ als Funktion von $V_{\mathrm{F}}$ aufgetragen ist.

Wie im folgenden gezeigt wird, ist die Abnahme der Beweglichkeit $\mu$ mit wachsender Vorspannung 
als ein Weglängeneffekt zu werten. Die Hall-Beweglichkeit bei $V_{\mathrm{F}}=0$ beträgt $342 \mathrm{~cm}^{2} / \mathrm{Vs}$.

Die relative Änderung des elektrischen Widerstandes $\Delta \varrho / \varrho_{0}$ in einem transversalen Magnetfeld $H$ ist gemäß der bei Anwesenheit von einer Art von Ladungsträgern und schwachen Magnetfeldern anwendbaren Theorie dem Produkt $\mu^{2} H^{2}$ proportional mit einem Proportionalitätsfaktor der Größenordnung 1. Die Beziehung ist bei den untersuchten Bikristallen im Temperaturbereich von $40-250{ }^{\circ} \mathrm{K}$ einigermaßen gut erfüllt (s. Abb. 5). Bei tiefen Temperaturen treten Anomalien auf, die hier nicht diskutiert werden sollen. Man wird also erwarten, daß auch die transversale magnetische Widerstandsänderung mit $H$ senkrecht zur Korngrenze bei $77^{\circ} \mathrm{K}$ mit zunehmender Spannung abnimmt. Daß dies der Fall ist, geht aus Abb. 10 hervor. Neben der relativen Änderung der transversalen magnetischen Widerstandsänderung ist $\left(\mu_{\mathrm{v}} / \mu_{0}\right)^{2}$ als Funktion von $V_{\mathrm{F}}$ aufgetragen. Es ist zwar keine quantitative Übereinstimmung vorhanden, immerhin aber eine qualitative.

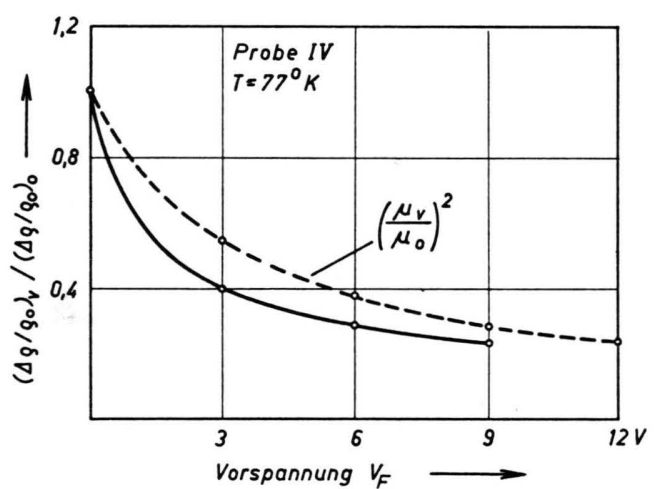

Abb. 10. Relative transversale magnetische Widerstandsänderung mit $H=10 \mathrm{kOe}$ senkrecht zur Korngrenze als Funktion einer symmetrischen Vorspannung (ausgezogene Kurve) und Quadrat der relativen Änderung der HALL-Beweglichkeit in

Abhängigkeit von der Vorspannung (gestrichelte Kurve).

An der Feldeffektprobe IV wurde bei $77^{\circ} \mathrm{K}$ eine kleine, aber meßbare longitudinale magnetische Widerstandsänderung gefunden. In einem Magnetfeld von $10 \mathrm{kOe}$ ist bei der Vorspannung 0 die relative Widerstandsänderung $\Delta \varrho / \varrho_{0}$ kleiner als $10^{-4}$. Aus Abb. 11 kann man entnehmen, daß $\Delta \varrho / \varrho_{0}$ proportional zu $H^{1,37}$ mit dem Magnetfeld anwächst. Während die transversale magnetische Widerstandsänderung ( $H \perp$ Korngrenze) beim Anlegen einer Feldspannung abnahm, nahm der Longitudinaleffekt zu. Dieser Befund kann z. Zt. nicht gedeutet werden. An an-

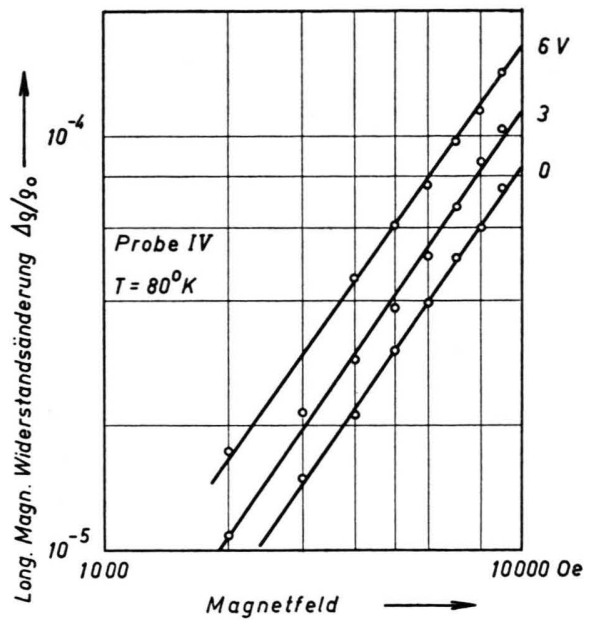

Abb. 11. Longitudinale magnetische Widerstandsänderung als Funktion des Magnetfeldes mit der Vorspannung als Parameter.

deren Bikristallproben wurde bei tiefen Temperaturen ein beträchtliches Ansteigen der longitudinalen magnetischen Widerstandsänderung gefunden. Bisher wurde für dieses Verhalten noch keine befriedigende Erklärung gefunden. Bei der longitudinalen magnetischen Widerstandsänderung in der Raumladungszone an einer Korngrenze hat man mit Sicherheit ein sehr komplexes Problem vor sich. Die Möglichkeit, diesen Effekt durch ein elektrisches Querfeld zu beeinflussen, erscheint interessant und es dürfte sinnvoll sein, ihn in Zukunft eingehender in dieser Weise zu studieren.

Einige Feldeffektmessungen wurden bei $4,2{ }^{\circ} \mathrm{K}$ ausgeführt. In Abb. 12 ist die relative Widerstandsänderung $R_{\mathrm{v}} / R_{0}$ von Probe IV beim Anlegen von symmetrischen und unsymmetrischen Vorspannun-

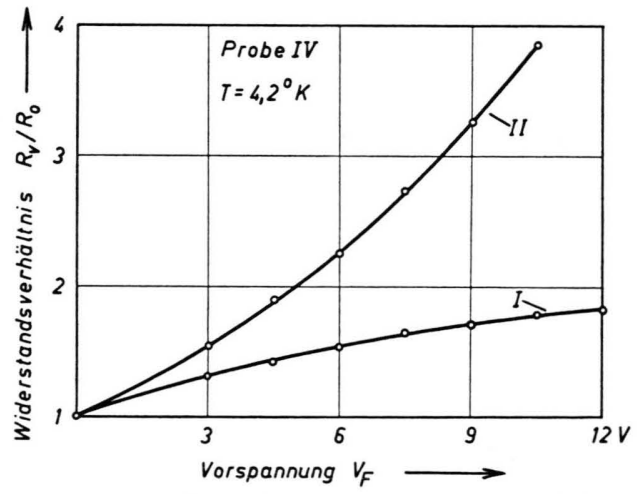

Abb. 12. Relative Änderung des elektrischen Widerstandes $R_{\mathrm{V}} / R_{0}$ der Raumladungszone als Funktion einer asymmetrischen (I) und einer symmetrischen Vorspannung (II) bei $T=4,2^{\circ} \mathrm{K}$. 
gen $V_{\mathrm{F}}$ aufgetragen. Man sieht, daß der Effekt größer ist als bei $77^{\circ} \mathrm{K}$. Die Widerstandserhöhung im Falle der parallel geschalteten Feldelektroden wächst stärker als linear mit der Vorspannung an. Während bei Messungen bei Stickstoff-Temperatur der Feldeffekt innerhalb der Fehlergrenzen von $\pm 2 \%$ von der Polarität des Stromes unabhängig war, traten bei $4,2^{\circ}$ und parallel geschalteten Feldeffektelektroden Unterschiede der Größenordnung 10\% auf. HaLL-Effekt-Messungen bei Helium-Temperaturen und angelegter Querspannung erwiesen sich als wesentlich schwieriger als solche bei $77^{\circ} \mathrm{K}$. Der Grund dafür sind eine größere überlagerte magnetische Widerstandsänderung und offenbar durch Feldsonden-Sperrströme hervorgerufene Widerstandsschwankungen der Probe. Wegen des hohen Widerstandes im Kristalläußeren können zu große Sperrströme die Meßergebnisse viel eher in unkontrollierter Weise beeinflussen als bei höheren Temperaturen. Da die galvanomagnetischen Effekte bei tiefen Temperaturen, insbesondere die magnetische Widerstandsänderung, ein bis jetzt noch nicht verständliches anomales Verhalten zeigen ${ }^{5}$, wurde auf mit dem Feldeffekt gekoppelte galvanomagnetische Messungen bei $4,2^{\circ} \mathrm{K}$ vorläufig verzichtet.

Die hier berichteten Ergebnisse für den Kristall IV, die bei $77^{\circ} \mathrm{K}$ gewonnen wurden, konnten durch Experimente mit der Probe N 8 verifiziert werden. Zwischen den Resultaten für die Abhängigkeit des Beweglichkeitsverhältnisses von $V_{\mathrm{F}}$ besteht quantitative Ubereinstimmung. Unterschiede in der transversalen magnetischen Widerstandsänderung als Funktion der Feldspannung sind unwesentlich. Damit ist erwiesen, daß die gewählte Elektrodenanordnung keinen Einfluß auf die Meßergebnisse hatte.

Die aus einem früher untersuchten $2 \Omega \mathrm{cm}$-Bikristall präparierte Probe I zeichnete sich durch eine besonders hohe $\mathrm{H}_{\mathrm{ALL}}$-Beweglichkeit von $1070 \mathrm{~cm}^{2}$ pro Vs bei $77^{\circ} \mathrm{K}$ aus. Der Anstieg des Hall-Koeffizienten $R^{*}$ war für symmetrisch und unsymmetrisch angelegte Vorspannungen gleich und betrug 16\% bei $V_{\mathrm{F}}=9 \mathrm{~V}$ relativ zu $V_{\mathrm{F}}=0$. Die prozentuale $\ddot{\mathrm{n}}$ derung der HALL-Beweglichkeit als Funktion von $V_{\mathrm{F}}$ ist in Abb. 13 dargestellt. Bei Parallelschaltung der Feldelektroden ist die Beweglichkeitsabnahme innerhalb weniger Prozente gleich der an Probe IV beobachteten, während bei einseitiger Vorspannung bei

18 J. J. Thomson, Proc. Cambr. Phil. Soc. 11, 120 [1901].

19 K. Fuchs, Proc. Cambridge Phil. Soc. 34, 100 [1938].

20 E. R. Andrew, Proc. Phys. Soc., London A 62, 77 [1949].
Probe I ein qualitativ gleicher, jedoch kleinerer Effekt auftritt. Insgesamt kann die Übereinstimmung der Ergebnisse für verschiedene Proben als befriedi-

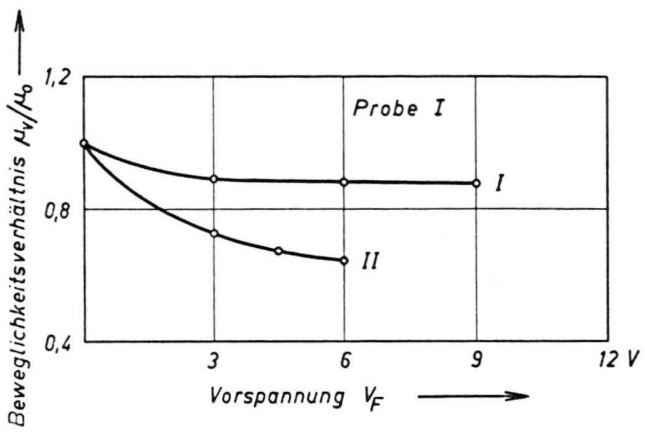

Abb. 13. Relative Änderung der HaLL-Beweglichkeit $\mu_{\mathrm{v}} / \mu_{0}$ als Funktion einer asymmetrischen (I) und einer symmetrischen Vorspannung (II) für Bikristallprobe I bei $77^{\circ} \mathrm{K}$.

gend angesehen werden. Bevor die physikalische Deutung der Ergebnisse diskutiert wird, soll ein kurzer Uberblick über theoretische Aspekte der elektrischen Leitfähigkeit in dünnen Raumladungsschichten gegeben werden.

\section{Deutung der Resultate}

Schon frühzeitig wurde bemerkt, daß dünne Metallschichten einen höheren spezifischen Widerstand haben können als kompaktes Material. Thomson ${ }^{18}$ deutete diese Erscheinung als einen Weglängeneffekt. Wenn die Dicke einer Metallschicht so gering ist, daß Zusammenstöße der Leitungselektronen mit der Oberfläche häufiger sind, als Zusammenstöße mit Phononen oder Verunreinigungen im Innern, reduziert diffuse Streuung an der Oberfläche die Beweglichkeit merklich. Diffuse Streuung bedeutet, daß für ein Elektron, das sich der Oberfläche nähert, die Streuung in jeden Raumwinkel des Halbraumes gleich wahrscheinlich ist. Eine elastische Streuung dagegen führt nur zu einer Vorzeichenumkehr der zur Oberfläche senkrechten Impulskomponente und läßt bei isotroper effektiver Masse den elektrischen Widerstand unverändert. Eine quantitative Theorie der Weglängeneffekte in dünnen Metallschichten, die von einer Lösung der BoLtzmanN-Gleichung ausging, wurde von FUCHS ${ }^{19}$ ausgearbeitet. Experimente ${ }^{20,} 21$ führten zu dem Schluß, daß in der Regel Zusammen-

${ }^{21}$ K. D. C. McDonald u. K. Sarginson, Proc. Roy. Soc., London A 203, 223 [1950]. 
stöße von Elektronen mit Metalloberflächen unelastisch sind. Eine Ausnahmestellung scheint Wismut ${ }^{22}$ einzunehmen, bei dem bisher nur elastische Oberflächenstreuung gefunden wurde.

Dünne Halbleiterschichten sollten sich ähnlich verhalten wie solche aus Metall. Es ist aber zu bedenken, daß die Situation in einem Halbleiter durch das Auftreten von Raumladungseffekten komplizierter ist. Weglängeneffekte in Germanium wurden vorwiegend an Oberflächeninversionsschichten untersucht. Bekanntlich sind Germaniumkristalle normalerweise mit einer dünnen Oxydschicht bedeckt, die Anlaß zu Oberflächenzuständen ${ }^{23}$ gibt. Damit sind lokalisierte Energiezustände an der Oberfläche des Kristalls gemeint. Eine Besetzung der Oberflächenzustände ändert die Leitfähigkeit der darunter liegenden Halbleiterzone. Negativ geladene Oberflächenzustände haben beispielsweise einer Oberflächenschicht aus n-Material Elektronen entzogen, es ist eine Verarmungsrandschicht entstanden. Die Elektronenverarmung kann so weit gehen, daß die Randschicht p-leitend wird. Man spricht dann von einer Inversionsschicht. In der Inversionsschicht herrscht ein ortsabhängiges elektrisches Feld, dessen Verlauf man durch eine Lösung der Porssonschen Gleichung ermitteln kann. Die Defektelektronen bewegen sich dann in einem Potentialtrog und stoßen bei hinreichend starker Inversion häufiger mit der Oberfläche zusammen als mit Phononen oder Störstellen. Nimmt man an, daß die Streuung an der Oberfläche diffus ist und diejenige am Potentialtrog elastisch (diese Annahme scheint realistisch zu sein), so besteht der Unterschied zu einer Metallschicht gleicher Dicke darin, daß die mittlere freie Weglänge bei ausschließlicher Oberflächenstreuung doppelt so groß ist. Schrieffer ${ }^{16}$ hat eine Theorie der Beweglichkeit von Ladungsträgern in dünnen Inversionsschichten entwickelt.

Bei Benutzung des exakten Raumladungspotentials ist die Lösung geschlossen nicht darstellbar. Für den hier verfolgten Zweck genügt es jedoch, eine Näherungslösung zu betrachten. $\mathrm{Zu}$ einer guten Abschätzung der Löcherbeweglichkeit in einem Potentialtrog kann man gelangen, wenn man eine konstante Feldstärke in der Raumladungszone voraussetzt. Es läßt sich zeigen, daß sich insbesondere für relativ große Feldstärken die exakte und die näherungsweise Lö-

22 A. N. Friedmann u. S. H. Koenig, IBM J. Res. Develop. 4, 158 [1960]. sung kaum unterscheiden. Bezeichnet man die effektive Beweglichkeit im Potentialtrog mit $\mu_{\text {eff }}$ und diejenige im Volumenmaterial mit $\mu_{\mathrm{b}}$, so ergibt $\operatorname{sich}^{16}$

$$
\begin{aligned}
\frac{\mu_{\mathrm{eff}}}{\mu_{\mathrm{b}}} & =1-\exp \left(\alpha^{2}\right)(1-\operatorname{erf} \alpha) \\
& =\frac{2}{\sqrt{\pi}} \alpha \quad \text { für } \alpha \ll 1
\end{aligned}
$$

mit $\alpha \equiv \frac{\sqrt{2 k T m^{*}}}{e \tau E}, \quad \operatorname{erf} \alpha \equiv \frac{2}{\sqrt{ } \pi} \int_{0}^{\alpha} \exp \left(-x^{2}\right) \mathrm{d} x$.

Es bedeuten: $k=$ Boltzmannsche Konstante, $T=\mathrm{abs}$. Temperatur, $m^{*}=$ effektive Masse des Defektelektrons, $e=$ Elementarladung, $E=$ elektrische Feldstärke in der Raumladungszone, $\tau=$ Relaxationszeit im Volumen.

Für kleine Feldstärken nähert sich die Beweglichkeit derjenigen im Volumenmaterial, bei hohen Feldern ist die effektive Beweglichkeit in der Raumladungszone der Feldstärke umgekehrt proportional. Die Ableitung setzt sphärische Energieflächen im $\boldsymbol{k}$-Raum $(\boldsymbol{k}=$ Wellenzahlvektor $)$ voraus sowie Anwendbarkeit der Boltzmann-Statistik.

Das über Oberflächen Gesagte trifft sinngemäß für Korngrenzen in Germanium zu. Die Versetzungen in der Berührungsebene der Bikristallhälften mit ihren Akzeptoreigenschaften entsprechen den Oberflächenzuständen. Die mechanische Fehlordnung im Innern der Korngrenze läßt die Annahme diffuser Streuung sinnvoll erscheinen; die Existenz einer Inversionszone in Bikristallen vom n-Typ ist experimentell bewiesen. Um die Theorie mit den Resultaten der ausgeführten Feldeffektexperimente vergleichen zu können, ist es noch erforderlich, die elektrische Feldstärke in der Korngrenzenzone als Funktion der angelegten Vorspannung zu berechnen. Derartige Rechnungen wurden von SCHRIEFFER ${ }^{16}$ und von Statz ${ }^{15}$ und Mitarbeitern ausgeführt. Die hier gegebene Darstellung schließt an die Arbeit von Statz et al. an. Zur Erläuterung möge Abb. 14 die-

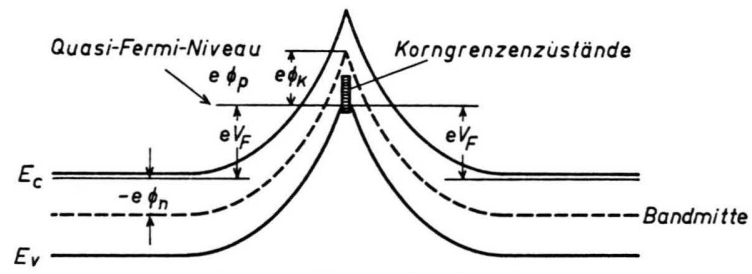

Abb. 14. Vereinfachtes Energiebänderschema einer symmetrisch vorgespannten Korngrenze. Die Bedeutung der Symbole ist aus dem Text zu ersehen.

23 Siehe z. B. Semiconductor Surface Physics, Ed. R. H. KInGston, University of Pennsylvania Press, Philadelphia 1957. 
nen, in der ein vereinfachtes Energiebänderschema einer symmetrisch vorgespannten Korngrenze aufgetragen ist. Da zwischen der p-leitenden Raumladungszone und dem n-leitenden Kristallgrundmaterial eine äußere Potentialdifferenz $V_{\mathrm{F}}$ besteht, existiert kein durchgehendes Fermi-Niveau. Es werden zwei Quasi-Fermi-Niveaus $e \Phi_{\mathrm{p}}$ und $-e \Phi_{\mathrm{n}}$ angenommen, die im p-bzw. n-Bereich konstant sind. Das Potential $\Phi$ wird auf ein Niveau bezogen, das praktisch mit der Bandmitte zusammenfällt und mit dem Fermi-Niveau im Eigenleitungsbereich identisch ist. Das Fermi-Niveau tief im Innern des n-Materials möge bei $-e \Phi_{\mathrm{n}}$ liegen (die Elektronenladung wird als $-e$ geschrieben) und die Bandaufwölbung in der Korngrenzenebene soll $e \Phi_{\mathrm{k}}$ betragen. Veränderungen der Bandstruktur im Innern der Korngrenze wurden außer acht gelassen. Man gewinnt die elektrische Feldstärke im Zentrum der Korngrenze durch Integration der Poissonschen Gleichung

$$
\frac{\mathrm{d}^{2} \Phi}{\mathrm{d} x^{2}}=-\frac{4 \pi e}{\varepsilon} \varrho(x),
$$

wo $\varrho(x)$ die Raumladungsdichte und $\varepsilon$ die Dielektrizitätskonstante bedeuten. Für die Raumladungsdichte in den drei Bereichen, die durch den Schnittpunkt der Quasi-Fermi-Niveaus mit der Referenzlinie für das Potential abgegrenzt wurden, erhält man folgende Werte:

$$
\varrho(x)=\left\{\begin{array}{c}
N_{\mathrm{D}}-n_{\mathrm{i}} \exp [e \Phi /(k T)] \\
\quad \text { für } \Phi_{\mathrm{n}} \geqq \Phi \geqq 0, \\
N_{\mathrm{D}} \text { für } 0>\Phi \geqq-V_{\mathrm{F}}, \\
N_{\mathrm{D}}+n_{\mathrm{i}} \exp \left[-e\left(\Phi+V_{\mathrm{F}}\right) /(k T)\right] \\
\quad \text { für }-V_{\mathrm{F}}>\Phi \geqq\left(-V_{\mathrm{F}}-\Phi_{\mathrm{k}}\right) ; \\
\quad n_{\mathrm{i}}=\text { Eigenleitungsdichte. }
\end{array}\right.
$$

Im ersten Bereich besteht die Raumladung aus ionisierten Donatoren mit der Dichte $N_{\mathrm{D}}$ und freien Elektronen (der Beitrag Defektelektronen kann vernachlässigt werden). Im zweiten Bereich sind nur ionisierte Donatoren vorhanden, und im dritten schließlich setzt sich die Raumladung aus geladenen Donatoren und freien Defektelektronen zusammen. Durch Änderung der Variablen und Integration von (2) über alle 3 Bereiche erhält man für die Feldstärke $E_{\mathrm{K}}$ im Zentrum der Korngrenze

$$
\begin{aligned}
E_{\mathrm{K}}=\left\{\frac { 8 \pi } { \varepsilon } \left[e N_{\mathrm{D}}\left(\left|V_{\mathrm{F}}\right|+\left|\Phi_{\mathrm{n}}\right|+\left|\Phi_{\mathrm{k}}\right|-\frac{k T}{e}\right)\right.\right. \\
\\
\left.\left.+e n_{\mathrm{i}} \frac{k T}{e} \exp \left\{e\left|\Phi_{\mathrm{k}}\right| /(k T)\right\}\right]\right\}^{1 / 2} .
\end{aligned}
$$

Die Rechnungen setzen die Anwendbarkeit der Boltzmannschen Statistik voraus. Die schwache Tem- peraturabhängigkeit der Leitfähigkeit der p-leitenden Bikristall-Raumladungszone und der nur wenig temperaturabhängige HaLL-Koeffizient $R^{*}$ lassen diese Voraussetzung zweifelhaft erscheinen. Andererseits sind die beobachteten Maxima in den Temperaturabhängigkeiten von $\sigma_{0}$ und $R^{*}$ ein Hinweis, daß nichtentartete Ladungsträger eine Rolle spielen. Überdies zeigt die Existenz eines Feldeffektes an den untersuchten Bikristallproben, daß die Dichte der freien Löcher in der Korngrenze nicht so groß sein kann, daß die eingebauten Donatoren zu vernachlässigen wären. Es erscheint daher gerechtfertigt, näherungsweise mit einer räumlich konstanten Feldstärke zu rechnen und das Produkt $n_{\mathrm{i}} \exp \left[e \Phi_{\mathrm{k}} /(k T)\right]$ durch eine mittlere Dichte der freien Defektelekronen $\bar{p}$ zu ersetzen. Weiterhin kann $k T / e$ gegenüber $\left|V_{\mathrm{F}}\right|+\left|\Phi_{\mathrm{n}}\right|+\left|\Phi_{\mathrm{k}}\right|$ vernachlässigt werden. Gemäß (1) ist die Beweglichkeit in einer Inversionsschicht bei hohen Feldstärken $E_{\mathrm{K}}$ (eine Voraussetzung, die in diesem Falle erfüllt ist) $E_{\mathrm{K}}$ umgekehrt proportional. Für das Beweglichkeitsverhältnis $\mu_{\mathrm{v}} / \mu_{0}$ erhält man dann

$\frac{\mu_{\mathrm{v}}}{\mu_{0}}=\frac{E_{\mathrm{k} 0}}{E_{\mathrm{kv}}}=\left[\frac{N_{\mathrm{D}}\left(\left|\Phi_{\mathrm{n}}\right|+\left|\Phi_{\mathrm{k}}\right|\right)+(k T / e) \bar{p}}{N_{\mathrm{D}}\left(\left|V_{\mathrm{F}}\right|+\left|\Phi_{\mathrm{n}}\right|+\left|\Phi_{\mathrm{k}}\right|\right)+(k T / e) \bar{p}}\right]^{1 / 2}$.

Es ist interessant, festzustellen, daß es gelang, die in Abb. 9 dargestellten experimentellen Daten für $\mu_{\mathrm{v}} / \mu_{0}$ bei symmetrisch vorgespannter Korngrenze mit einer Abweichung $<1 \%$ durch einen wie (4) gebauten Ausdruck

$$
\frac{\mu_{\mathrm{v}}}{\mu_{0}}=\left[\frac{1+0,35 \Phi}{1+0,35\left(\Phi+V_{\mathrm{F}}\right)}\right]^{1 / 2}
$$

mit $\Phi=0,73 \mathrm{~V}$ analytisch darzustellen. Die Anpassung ist ziemlich unempfindlich gegen Variationen von $\Phi$ in der Größenordnung $10 \%$, so daß sich aus den Meßdaten keine verläßlichen Informationen über die Lage der Fermi-Kante an der Korngrenze gewinnen lassen. Immerhin ergibt sich ein plausibler Wertbereich von $\Phi_{\mathrm{k}}$, wenn eine Breite der verbotenen Zone $0,732 \mathrm{eV}^{24}$ und ein Abstand zwischen Quasi-Fermi-Niveau im n-Material und der Unterkante des Leitungsbandes von $0,05 \mathrm{eV}$ zugrunde gelegt werden. Wegen der Unsicherheit von $\Phi_{\mathrm{k}}$ erscheint es nicht sinnvoll, die Dichte $p$ der freien Defektelektronen am Orte der Korngrenze nach der Beziehung $\quad p=n_{\mathrm{i}} \exp \left[e \Phi_{\mathrm{k}} /(k T)\right] \quad z u$ berechnen. Durch Vergleich von (4) mit (5) ermittelt man die

24 J. R. Haynes, M. Lax u. W. Flood, J. Phys. Chem. Solids 8, 392 [1959]. 
Beziehung $e N_{\mathrm{D}} /(\bar{p} k T)=0,35$ und kann damit die mittlere Dichte der freien Löcher abschätzen. $N_{\mathrm{D}}$ läßt sich aus dem spezifischen Widerstand des Kristallgrundmaterials zu $6 \cdot 10^{14} / \mathrm{cm}^{3}$ bestimmen. Damit ergibt sich für $\bar{p}$ bei $77^{\circ} \mathrm{K}$ ein Wert von $3,2 \cdot 10^{17} / \mathrm{cm}^{3}$.

Gl. (5) läßt sich auf den Fall der asymmetrisch vorgespannten Korngrenze ausdehnen, wenn man annimmt, daß die Beweglichkeit der nicht vorgespannten p-Zone unverändert bleibt und daß die beiden durch die Korngrenze getrennten stromtragenden Schichten unabhängig voneinander sind. Man erhält dann:

$$
\frac{\mu_{\mathrm{V}}}{\mu_{0}}=\frac{2(1+0,35 \Phi)^{1 / 2}}{(1+0,35 \Phi)^{1 / 2}+\left[1+0,35\left(\Phi+\left|V_{F}\right|\right)\right]^{1 / 2}} .
$$

Dieser Ausdruck führte nicht zu einer so guten Beschreibung der Meßwerte wie (5). Die Abweichung zwischen den nach Gl. (6) berechneten Punkten und den experimentellen Daten betrug ca. $2 \%$ bei $6 \mathrm{~V}$ und ca. $5 \%$ bei $9 \mathrm{~V}$ Vorspannung, so daß die Übereinstimmung nur qualitativen Charakter hat.

\section{Diskussion}

Es kann als sehr befriedigend angesehen werden, daß es möglich war, die experimentellen Ergebnisse an einen theoretisch abgeleiteten Ausdruck anzupassen, der von der Voraussetzung ausgeht, daß die Beweglichkeit der Ladungsträger in der Raumladungszone durch diffuse Streuung an der Korngrenze bestimmt wird. Damit dürfte der Beweis erbracht sein, daß bei den untersuchten Bikristallen Weglängeneffekte bei Stromtransport entlang der Korngrenze von Bedeutung sind.

Die nicht quantitative Wiedergabe der Meßwerte durch Gl. (6) bei unsymmetrischer Vorspannung ist verständlich, da ein Durchgreifen des angelegten Feldes in die nicht vorgespannte p-Zone außer Betracht blieb. Die quantitative Darstellbarkeit der Daten bei symmetrisch vorgespannter Korngrenze durch Gl. (5) sollte nicht überbewertet werden, da es sich um eine Näherungsformel handelt, die z. B. die Änderung der Löcherdichte mit der Vorspannung $V_{\mathrm{F}}$ nicht berücksichtigt.

Bei einer Defektelektronendichte von $3,2 \cdot 10^{17} / \mathrm{cm}^{3}$ beträgt die Entartungstemperatur $44^{\circ} \mathrm{K}$, unter Annahme einer effektiven Masse von $0,3 m_{0}$. Demzufolge würde bei $77^{\circ} \mathrm{K}$ noch keine Entartung vorliegen und die gute Wiedergabe der experimentellen
Daten durch eine Formel, die klassische Statistik voraussetzt, wäre nicht so überraschend. Es ist aber offensichtlich, daß eine mittlere Dichte von $3,2 \cdot 10^{17}$ pro $\mathrm{cm}^{3}$ mit den $\mathrm{H}_{\text {ALL }}$-Effekt-Daten nicht in Übereinstimmung ist. Aus der Feldstärke bei $V_{\mathrm{F}}=0$, die sich zu $2,48 \cdot 10^{4} \mathrm{~V} / \mathrm{cm}$ ergibt, kann man mittels der bekannten Beziehung $Q=[\varepsilon /(4 \pi)] E_{\mathrm{K}}$ die Zahl der Ladungen pro Flächeneinheit $Q=4,4 \cdot 10^{11} / \mathrm{cm}^{2}$ abschätzen. Aus HaLl-Messungen dagegen leitet man für die Dichte der freien Löcher einen Wert von $4,6 \cdot 10^{12} / \mathrm{cm}^{2}$ ab. Umgekehrt wurde mit Hilfe der Thomas-F Ermi-Methode unter Verwendung von Tieftemperatur-HaLL-Daten eine mittlere Löcherzahl pro Volumeneinheit von $4 \cdot 10^{18} / \mathrm{cm}^{3}$ errechnet ${ }^{12}$. Es erhebt sich die Frage nach der möglichen Ursache dieser Diskrepanz. Bisher wurde stillschweigend angenommen, da $\beta$ die Beziehung $R^{*}=1 /(p e)$ streng gültig sei. Es muß aber bezweifelt werden, daß dies der Fall ist. Das Maximum, das $R^{*}$ mit sinkender Temperatur durchläuft, ist ein Hinweis, daß die Verhältnisse nicht so einfach liegen. Es ist bekannt, daß der Hall-Koeffizient von Volumenmaterial (bei schwachen Magnetfeldern) vom Streumechanismus der Ladungsträger abhängt. Bei Anwendbarkeit der klassischen Statistik und Anwesenheit nur einer Art von Ladungsträgern beträgt der Proportionalitätsfaktor $f$ in der Beziehung zwischen HaLL-Koeffizient $R$ und Trägerdichte $R=f /(p e)$ bei Vorherrschen von Gitterstreuung $3 \pi / 8$, bei Dominieren von Streuung an ionisierten Störstellen $\frac{315}{51} \pi$. Im Falle hoher Ladungsträgerdichten, der die Verwendung der Fermi-Statistik erforderlich macht, hat $f$ den Wert 1.

Bisher wurde vorausgesetzt, daß die Ladungsträgerdichte ortsunabhängig ist. Das ist in der Raumladungszone an einer Korngrenze mit Sicherheit nicht der Fall. Komplikationen können auftreten, wenn mit dem Dichtegradienten senkrecht zur Korngrenze ein Gradient in der Beweglichkeit gekoppelt ist. Es soll hier nur daran erinnert werden, wie drastisch sich die Anwesenheit einer Minorität von "leichten“ Löchern, die eine etwa achtmal höhere Beweglichkeit haben als die „schweren“ Löcher, in den galvanomagnetischen Effekten ausdrückt. Die Verhältnisse in der Raumladungszone an einer Korngrenze sind wohl so verwickelt, daß es nicht gerechtfertigt erscheint, von einer so stark vereinfachten Theorie eine quantitative Beschreibung der experimentellen Ergebnisse zu erwarten. Zwar hat ZEMEL ${ }^{25}$ gezeigt, da $\beta$ das Produkt $R^{*} \sigma_{0}$ eine Beweglichkeit 25 J. N. Zemel, Phys. Rev. 112, 762 [1958]. 
ergibt, die (in dünnen Raumladungszonen) nicht mehr als $13 \%$ von der Leitfähigkeitsbeweglichkeit abweicht. Dieses Resultat beruht aber auf nicht ganz realistischen Annahmen. So wurde z. B. eine konstante Relaxationszeit benutzt.

Bei der Bildung des Verhältnisses $\mu_{\mathrm{v}} / \mu_{0}$ würde ein von $V_{F}$ unabhängiger Proportionalitätsfaktor in der Beziehung zwischen $R^{*}$ und $p$ herausfallen, so $\mathrm{da} ß$ die mitgeteilten Beweglichkeitsdaten von der angedeuteten Problematik frei sein sollten. Allerdings ist zu bedenken, daß das Verhältnis $N_{\mathrm{D}} / \bar{p}$ in (4) bestimmt wurde und daß $N_{\mathrm{D}}$ vom Volumenwert abweichen könnte. Es ist bekannt, daß sich in der Umgebung von Versetzungen CotTrelL-Atmosphären bilden können, d. h. daß sich (wegen des aufgeweiteten Gitters) Fremdatome bevorzugt abscheiden können. Eine um den Faktor 10 erhöhte Donatorenkonzentration in der Nähe der Korngrenze würde in der Tat Feldeffekt- und HALL-Daten miteinander verträglich machen. Es liegen auch Anzeichen vor, die auf eine größere Donatorenkonzentration in der Nachbarschaft der Versetzungen hindeuten. Gemäß den durch Gl. (5) dargestellten Daten bei symmetrisch vorgespannter Korngrenze ist das Verhältnis $N_{\mathrm{D}} / \bar{p}$ annähernd konstant. Da aber $\bar{p}$ (wegen des steigenden HALL-Koeffizienten $R^{*}$ ) mit zunehmender Vorspannung $V_{\mathrm{F}}$ abnimmt, sollte $N_{\mathrm{D}}$ sich ebenso verhalten. Da die zusätzliche Ionisation von Donatoren durch das angelegte Feld in relativ großer Entfernung von der Korngrenze erfolgt, wo die Donatorenkonzentration sich von derjenigen im Volumen nicht wesentlich unterscheiden dürfte, sollte bei Vorhandensein einer Cotrrell-Atmosphäre die mittlere Donatorenkonzentration mit wachsender Vorspannung $V_{\mathrm{F}}$ sinken. Gerade dies Verhalten war aus der Konstanz von $N_{\mathrm{D}} / \bar{p}$ zu fordern. Man müßte allerdings postulieren, daß die Störstellenkonzentration in der Nähe der Versetzungen von derjenigen im Volumen abhängt, denn Feldeffektversuche von Weinreich, Mataré und Wegener ${ }^{26}$ mit einseitig vorgespannten Bikristallen zeigten deutlich den Einfluß der Dotierung auf die Widerstandsänderung der Korngrenzenschicht. Die berichtete Leitfähigkeitsänderung eines $2,7 \Omega \mathrm{cm}$-Bikristalls stimmt mit den hier mitgeteilten etwa überein. Ein detaillierter Vergleich ist wegen der unterschiedlichen Versuchsbedin-

26 O. Weinreich, H. F. Mataré u. B. Reed, Solid State Physics in Electronics and Telecommunications 1, S. 97, Academic Press, London-New York 1960. gungen jedoch nicht möglich. WeInReich et al. führten den beobachteten Feldeffekt auf eine Änderung der Zahl der Ladungsträger zurück.

Unlängst haben Palmer, Morrison und DauenBAUGH ${ }^{27}$ von „channel“-Experimenten an reinen GeOberflächen, die durch Spalten von Kristallen im Ultrahochvakuum erzeugt werden, berichtet. Die Resultate wurden mit Hilfe der Ergebnisse von Schrieffer ${ }^{16}$ und Statz et al. ${ }^{15}$ analysiert. Die Autoren kommen zu dem Schluß, daß trotz Überschreitens des Anwendungsbereichs der benutzten Theorie (reine Oberflächen vom Germanium zeigen wie die hier untersuchten Bikristalle ein ziemlich temperaturunabhängiges Verhalten, was auf Entartung hindeutet) die Experimente befriedigend interpretiert werden können. Die Situation bei Bikristallen scheint analog zu sein.

Missman und Handler ${ }^{28}$ haben bei Zimmertemperatur die HaLL-Beweglichkeit in durch Ionenbeschuß gereinigten Germaniumoberflächen gemessen. Verschiedene Bandaufwölbungen - und damit unterschiedliche elektrische Feldstärken in der Oberflächenschicht - wurden durch Variation der Sauerstoffbedeckung hervorgerufen. Es ergab sich eine ausgeprägte Abnahme der Beweglichkeit mit zunehmender Bandaufwölbung, die jedoch geringer war als die von Schrieffers Theorie geforderte (bei Annahme diffuser Streuung).

Es wurde nicht versucht, mit Hilfe von (1) eine Beweglichkeit in der Korngrenze zu berechnen, da anzunehmen ist, daß die Relaxationszeit $\tau$ von Defektelektronen in der Korngrenzenzone geringer ist als diejenige im Inneren des Kristalls. Es erscheint nicht ausgeschlossen, daß die von Missman und Handler bemerkten Abweichungen von Schrieffers Theorie bei Benutzung einer kleineren Relaxationszeit verschwinden würden.

\section{Schlußfolgerung}

Messungen der mittleren HALL-Beweglichkeit in der p-leitenden Raumladungszone, welche an die Korngrenze der untersuchten Bikristalle anschließt, ergaben eine merkliche Abnahme der Beweglichkeit mit zunehmender Vorspannung der p-Zone gegenüber dem n-leitenden Kristalläußeren. Die Beweg-

27 D. R. Palmer, S. R. Morrison u. C. E. Dauenbaugh, Phys. Rev. 129, 608 [1963].

28 R. Missman u. P. Handler, J. Phys. Chem. Solids 8, 109 [1959]. 
lichkeitsabnahme ist bei symmetrisch vorgespannter Korngrenze größer als bei einseitigem Anlegen einer Vorspannung. Auch Messungen der transversalen magnetischen Widerstandsänderung führen zu dem Schluß, daß mit zunehmender Spannungsdifferenz zwischen Korngrenze und Kristall die Beweglichkeit reduziert wird. Die Meßresultate lassen sich zwanglos deuten, wenn man annimmt, daß die mittlere freie Weglänge der Defektelektronen vorwiegend durch unelastische Zusammenstöße mit der Korngrenze bestimmt wird; durch Anlegen einer Vorspannung wird die mittlere freie Weglänge reduziert. Die experimentellen Ergebnisse lassen sich befriedigend mit Hilfe einer vereinfachten Theorie beschreiben, die die Anwendbarkeit der BoltzmanNStatistik voraussetzt. Es war möglich, eine mittlere
Dichte $\bar{p}$ der freien Defektelektronen an der Korngrenze abzuschätzen. Der mittlere Wert von $\bar{p}$ liegt niedriger als ein aus HALL-Effekt-Daten bestimmter. Es erscheint nicht ausgeschlossen, daß der Unterschied wenigstens zum Teil dadurch bedingt wird, daß der Zusammenhang zwischen reduziertem HALLKoeffizienten und Flächendichte der Ladungsträger komplizierter ist als angenommen wurde.

Ein Teil der hier mitgeteilten Untersuchungen wurde an der University Illinois, Urbana, USA, ausgeführt. Für die Bereitstellung der Einrichtungen des Kältelaboratoriums der Physikalisch-Technischen Bundesanstalt danke ich Herrn Dr. W. RüHL. Den Herren Prof. J. Bardeen und Prof. M. Kohler bin ich zu Dank verpflichtet für das Interesse, das sie an dieser Arbeit bekundeten, sowie J. M. Driver, R. M. Work und H. J. Engelmann für ihre technische Assistenz.

\title{
Die Normalprozesse und ihr Einfluß auf die Gitterwärmeleitfähigkeit in Festkörpern mit Stufenversetzungen
}

\author{
Helmut Bross, Peter Gruner und Peter Kirschenmann \\ Institut für Theoretische und Angewandte Physik der Technischen Hochschule Stuttgart \\ und Max-Planck-Institut für Metallforschung Stuttgart \\ (Z. Naturforschg. 20 a, 1611-1625 [1965] ; eingegangen am 31. Juli 1965)
}

\begin{abstract}
Es wird ein Formalismus entwickelt, mit dem man den Einfluß der Wechselwirkung der Gitterschwingungen untereinander auf die Gitterwärmeleitfähigkeit zahlenmäßig behandeln kann. In der niedrigsten Ordnung in der Anharmonizität und der Störungsrechnung werden bei einem Streuprozeß zwei Phononen vernichtet und ein drittes erzeugt oder umgekehrt. Aus den Erhaltungssätzen für Energie und Impuls folgt, daß man zwei Arten von solchen Dreiphononenprozessen unterscheiden kann. Prozesse, bei denen die Energie aller beteiligten Phononen miteinander vergleichbar ist, und solche, bei denen die Energie zweier Phononen wesentlich größer als die des dritten Phonons ist. Es wird gezeigt, daß durch die zuletzt genannten Prozesse bei langen Wellen keine Divergenzen bei der Lösung der Boltzmans-Gleichung mehr auftreten können. Zur Lösung des Transportproblems wird ein Variationsverfahren benützt. Die dabei für Dreiphononenprozesse charakteristischen Größen werden berechnet. Dieses Verfahren gestattet in einfacher Weise das Zusammenwirken der Streuung der Gitterwellen aneinander und an statischen Gitterfehlern zu behandeln, das für die Einstellung des thermischen Gleichgewichts wichtig ist. Als Beispiel hierfür wird der Fall gerader Stufenversetzungen untersucht. Alle numerischen Rechnungen werden für Kupfer durchgeführt.
\end{abstract}

\section{Einleitung}

Seit den Überlegungen von Peierls ist das $\mathrm{Zu}$ standekommen der endlichen Gitterwärmeleitfähigkeit in einem Festkörper prinzipiell verstanden. Neben der Wechselwirkung der Gitterschwingungen mit den Leitungselektronen in Metallen führt vor allem ihre Wechselwirkung untereinander, die durch die Anharmonizitäten des Gitterpotentials bedingt ist, zu einem nichtverschwindenden Wärmewiderstand. In der niedersten Ordnung der Anharmonizität können zwei Streumechanismen unterschieden werden: 1 . Die Streuung der Gitterschwingungen an statischen
Gitterfehlern, bei der zwischen zwei verschiedenen Gitterschwingungen gleicher Frequenz ein Phonon ausgetauscht wird und 2. Drei-Phononen-Prozesse, bei denen zwei Gitterschwingungen jeweils ein Phonon an eine dritte Gitterschwingung abgeben oder bei denen der umgekehrte Vorgang abläuft. Bei einem solchen Prozeß müssen die Erhaltungssätze für den Quasiimpuls und die Energie

$$
\begin{aligned}
\mathfrak{f}+\mathfrak{f}^{\prime} & =\mathfrak{l}^{\prime \prime}+\mathfrak{\Re}^{(l)}, \\
\omega_{\mathfrak{t}}^{j}+\omega_{\mathfrak{k}^{\prime}}^{j^{\prime}} & =\omega_{\mathfrak{l}^{\prime \prime \prime}}^{j^{\prime \prime \prime}}
\end{aligned}
$$

erfüllt sein. Hierbei bezeichnet $\omega_{\ddagger}^{j}$ die Frequenz einer Gitterwelle mit Ausbreitungsvektor $\mathfrak{f}, j$ ihre Polari- 